\title{
Chancen und Grenzen der Evaluation informeller Bildungsprozesse von Kindern und Jugendlichen - Ergebnisse einer Evaluationssynthese mit dem Fokus auf Patenschaftsprojekte
}

\author{
Kurzfassung der mit dem DeGEval-Nachwuchspreis \\ ausgezeichneten Masterarbeit
}

\section{Julia Nick}

\section{Einleitung}

Overwien stellte bereits im Jahr 2006 (S. 58) fest, dass die begleitende Forschung in Bezug auf den Gegenstand informellen Lernens in Deutschland erst ,am Anfang“ steht. Folgt man vor diesem Hintergrund der Argumentation von Filsinger (2018: 782), kommt er auch über zehn Jahre später noch zu dem Schluss, dass „die Evaluation informellen Lernens [...] eine Entwicklungsaufgabe für die Evaluationsforschung [ist], die diese nur in Kooperation mit der (Grundlagen-)Forschung bewältigen kann". Insbesondere während der Evaluation von Patenschaftsprojekten wird häufig deutlich, dass diese Aufgabe auch aktuell auf der Agenda von Grundlagenund Evaluationsforschung stehen sollte, da bei der wissenschaftlichen Bearbeitung des Gegenstandes informeller Lernprozesse bestimmte Herausforderungen auftreten, die es zu bewältigen gilt. Der vorliegende Artikel soll in diesem Zusammenhang einen Versuch darstellen, ebendiese Herausforderungen in einem konkreten Handlungszusammenhang, nämlich eingebettet in Patenschaftsprojekte für Kinder und Jugendliche, sichtbar zu machen und Vorschläge für eine zielführende Evaluationspraxis in den Feldern soziale Dienstleistungen und Bildung aufzuzeigen. Hierzu wurden im Rahmen der Arbeit verschiedene Evaluationen von Patenschaftsprojekten betrachtet, die Erkenntnisse wurden innerhalb einer Evaluationssynthese zusammengeführt. Hense/Rädiker/Widmer/Böttcher (2013: 10) fassen die Zielsetzungen einer Meta-Analyse oder Evaluationssynthese wie folgt zusammen: „Evaluationssynthesen führen die Befunde von vorbestehenden Evaluationsstudien zu einer oder meh-

1 htw saar, Saarbrücken 
reren Maßnahmen oder zu einer Problematik mit einem qualitativen Zugang zusammen. Das Ziel der beiden Zugänge ist also der Erkenntnisgewinn auf der Ebene des Evaluations- oder Untersuchungsgegenstands und nicht, wie bei der Forschung über Evaluationen, auf der Ebene der Evaluation“. Der Fokus lag in diesem Fall auf der Begleitforschung des Projektes „Lernpaten Saar“ (vgl. ForBES 2017), da hierdurch eigene Forschungserfahrungen einbezogen werden konnten. Zusätzlich wurde das Patenschaftsprojekt an einer Kieler Schule (vgl. hierzu Selle 2016: 129ff.) und das Mentoring-Projekt „Balu und Du“ (vgl. Esch/Müller-Kohlenberg/Siebolds/Szczesny 2007 und Müller-Kohlenberg 2018) in die Betrachtung einbezogen.

\section{Theoriebezogene Überlegungen zu informellen Lernprozessen als Forschungsgegenstand}

Erste Hürden ergeben sich bei der Erforschung informeller Lern- und Bildungsprozesse bereits bei der Begriffsbestimmung, wodurch sich auch die systematische empirische Betrachtung erschwert (vgl. Filsinger 2018: 787). Darüber hinaus können weitere Herausforderungen identifiziert werden, die sowohl für die Grundlagen- als auch für die Evaluationsforschung relevant werden. Für Rauschenbach (2018) gibt es drei solcher Herausforderungen, die besonders prominent erscheinen. Zum einen stellt er fest, dass die Schwierigkeit besteht, nicht gesteuerte und somit informelle Lernprozesse - aufgrund ihrer Eigenschaft, immer und überall stattfinden zu können - während des Forschungsprozesses überhaupt beobachten zu können. Weiterhin merkt er an, dass die Feststellung von Kausalität von Lernergebnissen und den erfolgten Lernprozessen nur äußerst eingeschränkt vorgenommen werden kann. Gerade im Hinblick auf den Erwerb sozialer Kompetenzen beziehungsweise Soft Skills kann die Ursache für einen Zuwachs nur in Ansätzen festgestellt werden. Eine dritte Herausforderung wird auf die Umsetzung der Messbarkeit solcher Wissenszuwächse bezogen, die den Kriterien wissenschaftlicher Standards entsprechen würde (vgl. Rauschenbach 2018: 822). Thole (2018: 480) fasst diese Herausforderungen, bezogen auf Evaluationsforschung, wie folgt zusammen: „Insbesondere die jüngere, die Wirksamkeit konkreter Lehr- und Lernsituationen empirisch evaluierende Forschung stößt auf Schwierigkeiten, das artikulierte Wissen und Können nachweislich und belastbar auf konkrete Methoden des Lehrens und Weisen des Lernens zu beziehen". Diese Schwierigkeiten berücksichtigend, könnte ein Beitrag der Evaluationsforschung zwar weniger darin bestehen, informelle Lernprozesse als isoliertes Wirkungsphänomen zu betrachten, sondern vielmehr ihr Potenzial als Instrument zu nutzen, die Systematik des Vollzugs informellen Lernens in bestimmten Kontexten zu erfassen und angepasst an die umliegenden Strukturen zu bewerten (vgl. Filsinger 2018: 788). Die Forschenden müssen folglich in der Lage sein, die ablaufenden Prozesse systematisch aufzudecken und eine Möglichkeit zu finden, die darin stattfindenden informellen Lernprozesse abbildbar zu machen. Es kann somit festgehalten werden, dass informelle Lernprozesse durch ihr Charakteristikum, nicht steuerbar zu sein und im Alltag quasi unsichtbar abzulaufen, eine große Herausforderung für die empirische Betrachtung bilden. Dies ist nicht nur auf Fragestellungen 
der Grundlagenforschung, sondern auch auf Aufträge innerhalb der Evaluationsforschung übertragbar. Anhand der Ausführungen wird deutlich, dass diese Lernprozesse zwar nicht klassisch vermessen werden können, wohl aber über die kreative Anwendung und Kombination von Methoden zu erfassen und rekonstruierbar sind.

\section{Konsequenzen für den Umgang mit informellem Lernen als Forschungsgegenstand in der Evaluation}

Betrachtet man die gewonnenen Erkenntnisse in Bezug auf den Gegenstand informeller Lern- und Bildungsprozesse wird deutlich, dass eine Bestimmung nur im Rahmen der Beachtung ihres Eigensinns und unter Berücksichtigung der projektbezogenen Bedingungen gelingen kann. Um dies im Rahmen der (Auftrags-)Forschung gewährleisten zu können, erfordert es ein hohes Maß an Fachwissen seitens der Forschenden. Zusätzlich müssen diese darüber hinaus auch über eine hohe Sensibilität für die Zusammenhänge von lerntheoretischen Zugängen und Alltagsprozessen verfügen, um die Eigenart informellen Lernens und die daraus entstehenden Kompetenzgewinne überhaupt abbilden und bewerten zu können. Generell gestaltet sich eine Bewertung von Wirkungen, wie sie im Rahmen von Evaluationen erwartet wird, äußerst schwierig. Allerdings sollten bei der Konzeption und der Umsetzung solcher Vorhaben bestimmte Aspekte beachtet werden, deren Relevanz anhand der in dieser Synthese einbezogenen Projekte deutlich wurde. Zunächst kann - bezogen auf die Gelingensbedingungen von Forschungs- beziehungsweise Evaluationsprojekten, die informelle Lernprozesse als Teil des zu betrachtenden Gegenstands fokussieren - ganz allgemein festgestellt werden, dass diese eine differenzierte Betrachtung auf verschiedenen Ebenen und aus verschiedenen Blickwinkeln erfordern. Ein hiermit verbundener, offener Zugang zum Gegenstand wird, wie bereits erörtert, durch partizipative, responsive Evaluationsansätze gefördert, da die Projektbeteiligten hierbei als Expertinnen und Experten ihrer Lebenswelt, und somit der Projektumwelt, als wichtige Informationsquelle anerkannt werden. Diese Innenperspektive kann im Rahmen von Evaluationen wichtige Hinweise darauf geben, wie das Design einer Studie zielführend angelegt sein muss, um diejenigen Prozesse in den Fokus der Betrachtung zu rücken, die aus Sicht der Beteiligten relevant sind. Hierbei ist es wichtig, dass die durchführenden Evaluierenden dennoch auf ihr Erfahrungswissen aus anderen Evaluationen zurückgreifen, um auch Aspekte zu beleuchten, die den direkten Beteiligten aufgrund der eigenen Nähe zum Projekt möglicherweise verborgen bleiben. Im Rahmen der Evaluation informeller Lern- und Bildungsprozesse könnte es bereichernd sein, interdisziplinäre Evaluationsteams zu bilden, deren Angehörige unterschiedliche fachliche Schwerpunkte repräsentieren. Sowohl ein sozialwissenschaftlicher als auch ein pädagogischer und auch ein psychologischer Blick auf die Prozesse würden in diesem Zusammenhang einen Mehrwert bedeuten, der der wissenschaftlichen Herangehensweise mit Sicherheit zugutekäme. Um eine solche Erweiterung der Perspektiven ermöglichen zu können, sei erneut darauf hingewiesen, dass auch die Projektbeteiligten über ein Sonderwissen verfügen, das hierbei äußerst nützlich sein kann. Zudem ermöglicht die Bündelung der unter- 
schiedlichen Erklärungs- und Verstehensansätze verschiedener Disziplinen ein tieferes Verständnis über die Strukturen und Mechanismen von Projekten.

Bezüglich des Evaluationsdesigns kann grundsätzlich für ein gut konzipiertes Mixed-Methods-Design plädiert werden. Dieses sollte ein integriertes Modell quantitativer und qualitativer Erhebungsmethoden enthalten. Die Auswertung sowie die Ergebnisse müssen hierbei nicht separat nebeneinandergestellt werden, sondern können integriert erfolgen. Der Methodenpluralismus sowie die Nutzung der Stärken und Schwächen beider Herangehensweisen sind zur Betrachtung informeller Lernprozesse insofern nützlich, als dass zunächst beispielsweise mit einem Fragebogen identifiziert werden kann, welche Indikatoren im Rahmen der Wirkungsbewertung einbezogen werden sollten. Deren Einbindung und die damit verbundenen Prozesse können dann unter Einbezug qualitativer Zugänge sichtbar gemacht werden. Darüber hinaus sollten auch Daten in die Analyse einfließen, die nicht erst durch die Evaluation erhoben wurden und somit künstlich generiert wurden. Aufgrund der Schwierigkeit, im Bildungssektor klassische Experimente (RCTs) durchzuführen, käme als Bestandteil eines quasi-experimentellen Vorher-Nachher-Vergleichs beispielsweise eine Bestandsaufnahme der Noten einzelner Schülerinnen und Schüler in Betracht. Ergänzend wäre es möglich, die von Lehrenden durchgeführte Dokumentation über einzelne Kinder und Jugendliche zu nutzen, um Entwicklungsverläufe und (positive) Lernentwicklungen auch im Nachhinein noch rekonstruieren zu können. Ein weiterer Aspekt, der allgemein im Rahmen der Evaluation von Projekten bürgerschaftlichen Engagements berücksichtigt werden kann, ist die Einbindung der Ehrenamtlichen selbst. Bei jedem der innerhalb dieser Betrachtung berücksichtigten Projekte wurden diese im Rahmen von Schulungen und Qualifizierungsmaßnahmen auf ihre ehrenamtliche Tätigkeit vorbereitet. Neben organisatorischen Inhalten wurde ihnen hierbei auch Fachwissen aus dem Bereich der Pädagogik sowie der Sozialen Arbeit vermittelt. Bei Evaluationen, die ex ante - also schon vor Beginn oder spätestens bei der Implementierung der Maßnahme - einsetzen, sollte die Qualifizierung durch den Aspekt der Entwicklungsbeobachtung und -dokumentation erweitert werden. Hierdurch könnten über die Dauer der gesamten Patenschaft zu verschiedenen Zeitpunkten Daten erhoben werden, die im Rahmen der Evaluierung eine Betrachtung auf Längsschnittebene erlauben würde. Auch wenn der exakte Ausschluss von Störfaktoren hierbei nicht möglich ist, könnte aufgrund der Einschätzungen der Ehrenamtlichen rekonstruiert werden, ob die Entwicklungen auf die durchgeführte Maßnahme zurückzuführen sind. Ein gut geeignetes Instrument, um die Eindrücke der Patinnen und Paten kontinuierlich abzufragen, sind Tagebücher. Diese werden am besten digital zur Verfügung gestellt, sodass die Inhalte abgesichert sind und ein einfacher Zugriff möglich ist. Zusätzlich sollte, gerade im Bereich von Bildung und sozialer Dienstleistungen, stets auf eine transparente Kommunikation mit dem/der Auftraggebenden geachtet werden. Nur so kann gewährleistet werden, dass Missverständnisse bezüglich der Realisierbarkeit und dem Nutzen der Ergebnisse von Evaluationen minimiert werden. Hierbei ist wichtig, dass mit forschungsmethodischen Herausforderungen transparent umgegangen wird, so dass die Zielsetzungen des Vorhabens entsprechend angepasst werden können. 


\section{Fazit und Ausblick}

Während der Auseinandersetzung mit dem Stand der Forschung wurde deutlich, dass eine eindeutige begriffliche Erfassung von informellen Lern- und Bildungsprozessen nur sehr schwer möglich ist. Daher wurde eher der Zugang über die Charakteristika informellen Lernens gewählt, der im Rahmen des Gesamtvorhabens sinnvoll erscheint. Als ,informell` können demnach all solche Lernprozesse bezeichnet werden, die aus Sicht des Lernenden intuitiv und unbewusst in alltäglichen Situationen geschehen und aus Sicht des ,Lehrenden“ nicht durch die Schaffung zielgerichteter Lehr-Lern-Settings gesteuert werden können. Dennoch wird in Bezug auf verschiedene Handlungsfelder, wie beispielsweise der Offenen Kinder- und Jugendarbeit oder im Bereich der Ganztagsschule, ein Mitdenken von derart gelagerten Lernprozessen äußerst relevant, wenn es um die Konzeption von Angeboten geht. Hier besteht die Möglichkeit, sowohl die sozialen als auch die alltagspraktischen Kompetenzen von Kindern und Jugendlichen zu fördern. Des Weiteren ist der positive Einfluss auf Aspekte der Persönlichkeitsentwicklung relevant. Das Potenzial einer 1:1-Beziehung, die als Patenschaft gesteuert ist, kann aus wissenschaftlicher Perspektive sehr hoch eingeschätzt werden. Umso wichtiger ist es sowohl für die Grundlagen- als auch für die Auftragsforschung, intensiver an einem Wissenstransfer zu arbeiten und von den Erkenntnissen Anderer zu profitieren. Die Evaluationsforschung kann hier den Beitrag leisten, den Gegenstand innerhalb strukturierter Vorgänge zu beobachten, nachzuvollziehen und - als Konsequenz einer jeden Evaluierung - auch Gelingensbedingungen für die Projektdurchführung von Patenschaftsprogrammen zu generieren. Anhand der betrachteten Studien wurde deutlich, dass gut gewählte methodische Herangehensweisen durchaus belastbare Ergebnisse liefern können, die insbesondere auch für die Programmsteuerung und die Legitimation des Einsatzes finanzieller Ressourcen herangezogen werden können. Dies war unter anderem auch eine Zielsetzung des zum Gegenstand gemachten Projektes „Lernpaten Saar“. Hier konnten die Projektverantwortlichen nicht nur ein positives Feedback bezüglich der Umsetzung und Koordination erhalten, sondern auch die Rückmeldung bekommen, inwiefern das Programm die fokussierten Ziele erreicht und welche Impulse nötig werden, um die Durchführung zu verbessern. Im Rahmen der Synthese konnten Konsequenzen auf zwei verschiedenen Ebenen abgeleitet werden: Für den Gegenstand informellen Lernens ist es grundsätzlich relevant diesen - auch wenn keine einheitliche Definition vorliegt - im Rahmen jedes ähnlich angelegten Projektvorhabens für sich zu definieren und die vielfältigen Einflussfaktoren $\mathrm{zu}$ beachten. Für die Konzeption und die Durchführung von Evaluationsstudien im Bereich informeller Lernprozesse wurde deutlich, dass diese mit einem hohen Anspruch an die Evaluierenden verbunden sind, die über ein hohes Maß an fachlichem und methodischem Geschick verfügen müssen, um den Gegenstand angemessen $\mathrm{zu}$ erforschen. Insbesondere eine Vielfalt der Methoden erschien hierbei perspektivenreich, um die Komplexität informellen Lernens in Ansätzen erfassen zu können. Da für die Erörterung nur ein kleiner Bruchteil der existierenden Programme und dazugehörigen (Evaluations-)Studien in die Betrachtung einbezogen werden konnte, wäre es zielführend eine solche Betrachtung auszuweiten und systematisiert durchzuführen. Hierdurch könnte eine 
Art ,Best-Practice'-Leitfaden entstehen, der Evaluierende unterstützen kann, die sich im Rahmen von Aufträgen mit der Analyse von informellen Lern- und Bildungsprozessen auseinandersetzen. Darüber hinaus eröffnen sich aufgrund der gesammelten Erkenntnisse auch weitere Felder, die im Rahmen dieser Ausführung nicht berücksichtigt werden konnten. Neben klassischen Patenschaftsprojekten an Schulen, gibt es gerade unter dem Stichwort „Mentoring“ auch in hochschulischen und betrieblichen Bereichen viele Projekte, die von einer Betrachtung aus einer evaluativen Perspektive profitieren würden. Des Weiteren wurde der Fokus hier lediglich auf die betreuten Kinder und Jugendlichen gelegt. Allerdings sollte - und wurde beispielsweise im Rahmen der Analysen des Programmes „Balu und Du“ - auch der Pate oder die Patin nicht außer Acht gelassen, wenn es um die Frage nach Wirkungen geht. Auch wenn diese nicht die fokussierte Zielgruppe der Projekte sind, finden auch bei ihnen (informelle) Lernprozesse statt, die bei der Forderung nach lebenslangem Lernen und dem Wirken von bürgerschaftlichem Engagement möglicherweise häufiger in Vergessenheit geraten. Abschließend kann konstatiert werden, dass die empirische und evaluationspraktische Auseinandersetzung mit informellen Lern- und Bildungsprozessen sich als ein vielfältiges zukunftsfähiges Feld gestaltet, das noch einige Möglichkeiten und Potenziale bildet, sich dezidiert mit diesen Gegenstandsbereichen auseinanderzusetzen.

\section{Literatur}

Esch, Dominik/Müller-Kohlenberg, Hildegard/Siebolds, Martina/Szczesny, Michael (2007): „Balu und Du“. Ein Mentorenprojekt für benachteiligte Kinder im Grundschulalter. In: Lernbegleitung und Patenschaften. Reflexive Fallarbeit in der universitären Lehrerausbildung. Bad Heilbrunn: Klinkhardt, S. 132-144.

Filsinger, Dieter (2018): Evaluationsforschung und informelles Lernen. In: Harring, Marius/Witte, Matthias D./Burger, Timo (Hg.): Handbuch informelles Lernen. Interdisziplinäre und internationale Perspektiven. Weinheim und Basel: Beltz/Juventa, S. 782-800 (2. Aufl.).

ForBES (2017): Pilotphase des Projektes „Lernpaten Saar“. Abschlussbericht der wissenschaftlichen Begleitung (Evaluationsbericht). Unveröffentlichtes Dokument.

Hense, Jan/Rädiker, Stefan/Widmer, Thomas/Böttcher, Wolfgang (2013): Notwendigkeit und Potenzial einer Forschung über Evaluation. In: Hense, Jan/Rädiker, Stefan/Widmer, Thomas/Böttcher, Wolfgang (Hg.): Forschung über Evaluation. Bedingungen, Prozesse und Wirkungen. Münster: Waxmann, S. 7-20.

Müller-Kohlenberg, Hildegard (2018): Handbuch Mentoring für Grundschulkinder. Balu und Du. 53 Bausteine für Theorie und Praxis. Ibbenbüren: Münstermann.

Overwien, Bernd (2006): Informelles Lernen - zum Stand der internationalen Diskussion. In: Rauschenbach, Thomas/Düx, Wiebken/Sass, Erich (Hg.): Informelles Lernen im Jugendalter. Vernachlässigte Dimensionen der Bildungsdebatte. Weinheim/München: Juventa, S. 35-62.

Rauschenbach, Thomas (2018): Informelles Lernen - Bilanz und Perspektiven. In: Harring, Marius/ Witte, Matthias D./Burger, Timo (Hg.): Handbuch informelles Lernen. Interdisziplinäre und internationale Perspektiven. Weinheim/Basel: Beltz/Juventa, S. 817-830 (2. Aufl.).

Selle, Ulrich (2016): Patenschaften bei auffälligem Verhalten. Ein Projekt für Risikokinder in der weiterführenden Schule. Weinheim/Basel: Beltz/Juventa.

Thole, Werner (2018): Non-formales und informelles Lernen in der Kinder- und Jugendhilfe. In: Harring, Marius/Witte, Matthias D./Burger, Timo (Hg.): Handbuch informelles Lernen. Interdisziplinäre und internationale Perspektiven. Weinheim/Basel: Beltz/Juventa, S. 479-500. 